\title{
Scattering Theory and Quadratic Forms: On a Theorem of Schechter *
}

\author{
B. Simon $\star \star$ \\ Department of Physics, Yeshiva University, New York, NY 10033, USA
}

\begin{abstract}
We show how to extend Cook's method to a class of pairs whose difference is only a quadratic form.
\end{abstract}

This note is connected with the existence question for generalized wave operators

$$
\Omega^{ \pm}(A, B)=\underset{t \rightarrow \mp \infty}{S-\lim _{t \rightarrow \infty}} e^{i t A} e^{-i t B} P_{a c}(B),
$$

where $A$ and $B$ are self-adjoint operators on a Hilbert space, $\mathscr{H}$, and $P_{a c}(B)$ is the projection onto $\mathscr{H}_{a c}(B)$, the set of vectorswhose spectral measure relative to $B$ is absolutely continuous with respect to Lebesgue measure. Nearly twenty years ago, Cook [2] proved a result whose abstract form says

Theorem 1. Let $A, B$ be self-adjoint operators and suppose that there is a subset $\mathscr{D}$ of $D(B)$ dense in $\mathscr{H}_{a c}(B)$ so that for any $\varphi \in \mathscr{D}$, there is a $T_{0}$ with $e^{i t B} \varphi \in D(A)$ for $|t|>T_{0}$ and

$$
\int_{|t| \geqq T_{0}}\left\|(B-A) e^{i t B}\right\| d t<\infty .
$$

Then $\Omega^{ \pm}(A, B)$ exist.

Cook's theorem is widely applicible (see e.g. [6]) and it has the advantage of having an extremely simple proof. As regards existence alone, its main disadvantage is that it does not accomodate operators defined as sum of forms, e.g. $B=-\Delta$, $A=-\Delta+V$. If $(1+|x|)^{+1+\varepsilon} V \in L^{3 / 2}\left(R^{3}\right)+L^{\infty}\left(R^{3}\right)$, one can define $A$ as a form sum and expects $\Omega^{ \pm}(A, B)$ to exist. Indeed, this has been proven [5], but only by developing the rather elaborate machinery of smoothness [4] and weighted $L^{2}$ estimates $[1,3]$ and the proof doesn't work in the multiparticle case. For many years, there have been no improvement in results based on an estimate like (1), but Schechter [7] has recently proven:

* $\quad$ Research supported by U.S. National Science Foundation under Grants MPS-75-11864

$\star \star$ On leave from Depts. of Mathematics and Physics, Princeton University, Princeton, NY 08540 , USA 
Theorem 2. Let $A$ and $B$ be self-adjoint operators on a Hilbert space $\mathscr{H}$. Let $C, D$, be operators from $\mathscr{H}$ to another Hilbert space $\mathscr{K}$ so that $C$ is relatively $A$ bounded and so that $A=B+C^{*} D$ in the sense that

$$
(A \varphi, \psi)=(\varphi, B \psi)+(C \varphi, D \psi)
$$

for all $\varphi \in D(A), \psi \in D(B)$. Suppose that there is a dense set $\mathscr{D}$ of $\mathscr{H}_{a c}(B)$ so that for any $\varphi \in \mathscr{D}$, there is $T_{0}$ with $e^{-i t B} \varphi \in D(D)$ for $|t|>T_{0}$ and

$$
\int_{|t|>T_{0}}\left\|D e^{-i B t} \varphi\right\| d t<\infty .
$$

Then $\Omega \pm(A, B)$ exist.

While Schechter's result is quite pleasing, it has the disadvantage of having a proof without the simplicity of Cook's method. It is our purpose here to show how to prove a result closely related to Schechter's using a small modification of Cook's original proof.

Theorem 3. Let $A$ and $B$ be self-adjoint operators on a Hilbert space $\mathscr{H}$ with $D\left(|A|^{1 / 2}\right)$ $=D\left(|B|^{1 / 2}\right)$. Suppose that $C$ and $D$ are operators from $\mathscr{H}$ to a Hilbert space $\mathscr{K}$ and that there is a dense set $\mathscr{D}$ in $\mathscr{H}_{a c}(B) \cap D(B)^{1 / 2}$ so that

(i) $C$ is $|B|^{1 / 2}$ relatively bounded.

(ii) For each $\varphi \in \mathscr{D}$, there is a $T_{0}$ so that for $|t|>T_{0}, e^{-i B t} \varphi \in D(D)$ and for any $\psi \in D\left(|B|^{1 / 2}\right)$ :

$$
\begin{aligned}
& \left(\psi, A e^{-i B t} \varphi\right)=\left(\psi, B e^{-i B t} \varphi\right)+\left(C \psi, D e^{-i B t} \varphi\right) . \\
& \text { (iii) } \int_{|t|>T_{0}}\left\|D e^{-i B t} \varphi\right\| d t<\infty .
\end{aligned}
$$

Then $\Omega^{ \pm}(A, B)$ exist.

Before proving this theorem, we note that it is "essentially" a specialization of Schechter's theorem although it will accommodate most practical situations where Theorem 2 is applicable. We mention:

Example. If $V=V_{1}+V_{2}$ where $V_{1} \in L^{3 / 2} \cap L^{3 / 2-\varepsilon}\left(\mathbb{R}^{3}\right)$, and $W=V_{2}(1+|x|)^{1+\varepsilon} \in L^{3 / 2}$ $+L^{\infty}$, then Theorem 3 shows that $\Omega^{ \pm}(-\Delta+V-\Delta)$ exist and generally is applicable to the multiparticle situation with these potentials. For let $C_{1}, C_{2}, D_{1}, D_{2}: \mathscr{H} \rightarrow \mathscr{H}$ by $C_{1}=\left|V_{1}\right|^{1 / 2}, D_{1}=V_{1} /\left|V_{1}\right|^{1 / 2}, C_{2}=W /|W|^{1 / 2}, D_{2}|W|^{1 / 2}(1+|x|)^{-1-\varepsilon}$. Since $V$ is $-\Delta$ form-bounded with relative form bound zero, $Q(-\Delta+V)=Q(-\Delta)$. Letting $\mathscr{D}$ $=\mathscr{H}\left(\mathbb{R}^{3}\right) \mathscr{K}=\mathscr{H} \oplus \mathscr{H}$ and $C=C_{1} \oplus C_{2} ; D=D_{1} \oplus D_{2}$, (i), (ii), (iii) hold. To check (iii) we note that $\int_{|t| \geqq 1}\left\|D_{1} e^{-i B t} \varphi\right\| d t<\infty$ by a standard argument ([6]) since $D_{1}$ is multiplication by a function in $L^{3-\delta}$. Moreover, since $|W|^{1 / 2}(-\Delta+1)^{-1}$ is bounded and $\varrho=(1+|x|)^{-1-\varepsilon}$ has $\varrho, \nabla \varrho, \Delta \varrho \in L^{3-\delta}$

$$
\begin{aligned}
& \left\|D_{2} e^{i t \Delta} \varphi\right\| \leqq \text { const }\left\|(-\Delta+1) \varrho e^{i t \Delta} \varphi\right\| \\
& \quad \leqq \operatorname{const}\left(\left\|\varrho e^{i t \Delta}(-\Delta+1) \varphi\right\|+\left\|(\Delta \varrho) e^{i t \Delta} \varphi\right\|+2\left\|\nabla \varrho \cdot e^{i t \Delta} \nabla \varphi\right\|\right.
\end{aligned}
$$

is in $L^{1}$. 
Proof of Theorem 3. Let $W(t)=e^{i A t} e^{-i B t}$. As usual [2], it suffices to prove that $\|(W(t)$ $-W(s)) \varphi \|^{2} \rightarrow 0$ as $t, s \rightarrow \infty$ (or $-\infty$ ), or equivalently that $(W(t) \varphi,(W(t)-W(s) \varphi) \rightarrow 0$ as $s, t \rightarrow \infty$. Now norm $D\left(|B|^{1 / 2}\right)$ with the norm $\left(\left\||B|^{1 / 2} \varphi\right\|+\|\varphi\|\right) \equiv\|\varphi \varphi\|$. Clearly $e^{-i t B}$ is an isometry in $\|\varphi\| \|$. Since $\|\mid \cdot\|$ is equivalent to the norm $\left\||A|^{1 / 2} \varphi\right\|+\|\varphi\|$ (by the closed graph theorem) $e^{-i t A}$ is bounded in ||$\cdot|| \mid$ uniformly in $t$.

$$
(W(t) \varphi,(W(t)-W(s)) \varphi)=\int_{s}^{t}\left(W(t) \varphi, e^{i u A} i(A-B) e^{-i u B} \varphi\right)
$$

so by (ii) for $s, t>T_{0}$ and say $t>s$

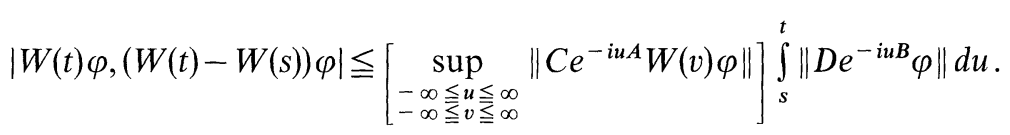

Now, since the maps $e^{-i u A} W(v)$ are uniformly bounded from $D\left(|B|^{1 / 2}\right)$ to itself $\varphi \in D\left(|B|^{1 / 2}\right)$ and $C$ is bounded from $D\left(|B|^{1 / 2}\right)$ to $\mathscr{K}$, the sup is finite. By (iii), the integral goes to zero if $s, t \rightarrow \infty$.

\section{References}

1. Agmon, S. : Spectral properties of Schrödinger operators and scattering theory. Ann. Scu. Norm. Sup. Pisa

2. Cook,J.: Convergence of the Møller wave matrix. J. Math. Phys. 36, 82-87 (1957)

3. Kuroda, S. : Scattering theory and differential operators, I, II. J. Math. Soc. Japan 25, 75-104, 222 234 (1973)

4. Lavine, R.: Commutators and scattering theory, II. A class of one body problems. Ind. J. Math. 21, $643-656(1972)$

5. Lavine, R.: Absolute continuity of positive spectrum for Schrödinger operators with long range potentials. J. Func. Anal. 12, 30-54 (1973)

6. Reed, M., Simon, B.: Methods of modern mathematical physics. III. Scattering theory. New YorkLondon: Academic Press 1978

7. Schechter,M.: A new criterion for scattering theory. Yeshiva Preprint

Communicated by W. Hunziker

Received November 25, 1976 
